Ulrike Ehmig, Konrad F. Zawadzki ${ }^{1}$

\title{
Anathema in lateinischen Inschriften
}

\section{Einleitung}

Die Auseinandersetzung mit dem spätantik-frühmittelalterlichen epigraphischen Gebrauch der Beschwörungsformeln adiuro und coniuro zeigt, dass diese in ganz spezifischen Medien und Kontexten zum Einsatz kamen. Zum einen tritt adiuro in Fluchtafeln auf, mit denen im 3. und 4. Jahrhundert n.Chr. in Nordafrika Gegner bei Wagenrennen am Sieg gehindert werden sollten. Beschworen wurden hierbei die Dämonen von Verstorbenen mit dem Ziel, gegnerische Lenker und Pferde am Lauf zu hindern. Zum anderen sind die Begriffe charakteristisch für christliche, vor allem in Rom und Italien konzentrierte Grabinschriften des späten 4. bis frühen 7. Jahrhunderts. In den betreffenden Epitaphen sind adiuro und coniuro jeweils Kern einer Grabschutzformel. Beschworen wurden die Leser der Inschriften, mitunter aber auch alle Christen, mit dem Ziel, das Grab vor einer Schändung zu schützen. Niemand sollte das Grab öffnen, die Totenruhe sollte nicht gestört, der Leichnam nicht entfernt und keine spätere Bestattung im selben Grab vorgenommen werden ${ }^{2}$.

1 Ulrike Ehmig - Dr. habil., Leiterin der Arbeitsstelle „Corpus Inscriptionum Latinarum“, Berlin-Brandenburgische Akademie der Wissenschaften Berlin, Deutschland; E-Mail: ulrike.ehmig@bbaw.de, ORCID: 0000-0003-1560-9022. Konrad F. Zawadzki - Dr., Leiter des DFG-Projekts „Der Kommentar Cyrills von Alexandrien zum 2. Korintherbrief. Einleitung, kritischer Text, Übersetzung, Einzelanalyse“, Theologische Fakultät Trier, Deutschland; E-Mail: zawadzki@uni-trier.de, ORCID: 0000-0003-0638-7034.

2 Unter dem Titel „Ich beschwöre... Zur epigraphischen Evidenz von ó $\rho$ kí $\omega$ und adiuro zwischen Antike und Hohem Mittelalter" ist eine gemeinsame Studie von Ulrike 
Nicht selten drohten die Grabstifter prophylaktisch abschreckend mit Sanktionen, falls den Anweisungen keine Folge geleistet würde. Neben Entschädigungen in Geld und Edelmetall, die schon bei paganen Grabinschriften seit Jahrhunderten üblich waren $^{3}$, belegte man in frühchristlicher Zeit potenzielle Grabschänder mit Flüchen, die dem Alten und Neuen Testament entstammten ${ }^{4}$. Geläufig war die Verwünschung zu einem Schicksal, wie es der Verräter Judas gefunden hatte. Eine Inschrift aus Santa Caecilia in Trastevere in Rom ${ }^{5}$ kombiniert diese mit einem zweiten Fluch, nämlich der Lepra des Syrers Naaman, und verhängt schließlich das Anathem als ewige Verdammnis über jene, die die Grabesruhe stören würden.

In den vergangenen Jahren fand eine intensive Auseinandersetzung mit Wesen und Entwicklung des Anathems auf der Grundlage der Schriften der frühen Christen statt ${ }^{6}$. Die epigraphische Evidenz des Begriffs wurde dabei noch nicht berücksichtigt. Die lateinischen Inschriften, die den Begriff anathema enthalten, eröffnen aber nicht nur eine weitere zeitliche und mediale Dimension des betreffenden Phänomens, sondern lenken den Blick auch auf andere Personengruppen und Verwendungs- und Bedeutungskontexte des Terminus. Vor diesem

Ehmig (Berlin), Juraj Franek, Daniela Urbanová (beide Brünn) und Konrad Knauber (Heidelberg) in Arbeit.

3 Dazu generell J.S Creaghan, Violatio sepulcri. An Epigraphical Study, Princeton 1951; J. Iluk, Amendes sépulcrales dans les épitaphes de l'époque de l'Empire Romain, Danzig 2013 und die bei U. Ehmig, Ausschlußverfahren. Eine Gruppe italischer Grabinschriften als Beispiel sozialer Überassimilierung in der römischen Kaiserzeit, „Epigraphica” 77 (2015) 193-205, [hier:] 198 Anm. 18 angeführten Werke.

4 Grundlegend dazu W. Speyer, Fluch, RACh VII, 1169-1288, [hier:] 1263-2165. Zum Zitat von Bibelstellen in der Epigraphik des lateinischen Westens C. Pietri, La Bible dans l'épigraphie de l'Occident latin, in: Le monde latin antique de la Bible, hg. von J. Fontaine - C. Pietri, Paris 1985, 189-205; vgl. auch M.T. Muñoz García de Iturrospe, Tradición formular y literaria en los epitafios latinos de la Hispania cristiana, (Veleia Series Minor 7), Vitoria Gasteiz 1995, 281-298.

5 ILCV 3866 = ICaRoma 120. Die Abkürzungen der epigraphischen Editionen folgen der betreffenden Liste in der Epigraphik-Datenbank Clauss Slaby EDCS unter http:// db.edcs.eu/epigr/hinweise/abkuerz.html (letzter Aufruf 10.5.2018). Anstelle von BE steht hier Bibl. Epigr.

6 K.F. Zawadzki, Die Anfänge des "Anathema“ in der Urkirche. Teil 1: Status quaestionis, VoxP 28 (2008) 1323-1334; K.F. Zawadzki, Die Anfänge des „Anathema“ in der Urkirche. Teil 2: Anhaltspunkte für das Anathem im Neuen Testament, VoxP 29 (2009) 495-520; K.F. Zawadzki, Die Anfänge des „Anathema" in der Urkirche. Teil 3: Anathematisierungspraxis in den Schriften der Apostolischen Väter und im apokryphen Korintherbrief, VoxP 30 (2010) 721-766; grundlegend auch schon K. Hofmann, Anathema, RACh I, 427-430. 
Hintergrund werden hier die lateinischen epigraphischen Zeugnisse für anathema vorgestellt, besprochen und die Beobachtungen den bisher anhand der frühchristlichen Schriftquellen ermittelten Ergebnisse gegenübergestellt?

\section{Vorstellung der lateinischen Inschriften mit dem Begriff anathema}

Insgesamt liegen elf einschlägige Inschriften vor ${ }^{8}$. Im Einzelnen handelt es sich um eine Bauinschrift aus Rom (1) sowie neun Epitaphe, von denen vier ebenfalls aus Rom stammen (2-5) und die übrigen Einzelstücke von der iberischen Halbinsel $(6,7)$, aus Aquitanien (8), der Gallia Narbonensis (9) und der Lugdunensis (10) darstellen. Die Inschrift aus Nordafrika (11) ist ebenfalls zwar auf einem - wesentlich älteren - Grabstein angebracht, ihre Funktion jedoch bleibt unklar. Ungeachtet dieses speziellen Falls zielt die Nennung von anathema in allen Inschriften als Drohung auf eine prophylaktische Schadensabwehr ab: Aus dem (geweihten) Wasser, das allen (nach Gott) Dürstenden zur Verfügung steht, soll kein Gewinn gezogen werden; Bauten sollen nicht zerstört und vor allem Gräber nicht geschändet werden. Im letztgenannten Fall soll damit konkret vor allem unerlaubten Nachbestattungen vorgebeugt werden'.

7 Die Studie beschränkt sich auf die lateinischen Zeugnisse, die explizit von anathema sprechen. Inschriften mit synonym verwendeten Begriffen wie maledictio - ILCV 3852 (Interamna Lirenas): Maledictione $D(e) i(h)$ avea $(t) / q(u i)$ istu $(m)$ sepulcrom misere / excepto Ursu et muliere sua - oder Umschreibungen ohne Nennung des hier interessierenden Terminus bleiben einer späteren Analyse vorbehalten. Einführend in die generelle Praxis der Anathematisierung in der frühchristlichen Epigraphik mit einzelnen Beispielen insbesondere aus dem griechischsprachigen Bereich C.M. Kaufmann, Handbuch der altchristlichen Epigraphik, Freiburg 1917, 152-160.

$8 \mathrm{Zu}$ Details siehe die nachfolgende Vorstellung der einschlägigen Inschriften.

9 Die Maßnahmen zum Grabschutz in christlichem Kontext spiegeln auch die in der betreffenden Zeit immer wichtiger werdende Diskussion und Sorge um ein intaktes Grab und einen ungestörten Leichnam als Voraussetzungen für eine körperliche Auferstehung, dazu W. Speyer, Fluch; Hausmair, Am Rande des Grabs. Todeskonzepte und Bestattungsritual in der frühmittelalterlichen Alamannia, Leiden 2015, 109-110; C.W. Bynum, The Resurrection of the Body in Western Christianity 200-1336 (Lectures on the History of Religions 15), New York 1995, 86-93; sowie zur mittelalterlichen und späteren Diskussion vgl. C.W. Bynum, Material Continuity, Personal Survival, and the 


\subsection{ILCV 784 (em.)}

Fund-/Aufbewahrungsort: Roma/Rom, in den Weingärten jenseits des Tibers in einem Brunnen; ehemals zu einer Kirche des Heiligen Marcus in Rom gehörig

Monument/Datierung: 7./8.Jh.?

$\mathrm{D}(\mathrm{e})$ don(is) $\mathrm{D}(\mathrm{e}) \mathrm{i}$ et $\mathrm{s}(\mathrm{an}) \mathrm{c}(\mathrm{t}) \mathrm{i}$ Marci / Ioh(annes) pr(es)b(yter) fier(i) (r)ogabit / omne(s) sitiente(s) venite be/nite ad aqua(m) et si quis $\mathrm{d}(\mathrm{e})$ ista / aqua pretio tuleri(t) / anathema sit

Aus den Gaben Gottes und des Heiligen Marcus veranlasste (dies) der Presbyter Iohannes; alle Dürstenden kommt, kommt zum Wasser, und wenn jemand von diesem Wasser Gewinn zieht, sei er mit dem Anathem belegt.

Kommentar: Bauinschrift eines Brunnens? ${ }^{10}$ Da keine weiteren Informationen zum schrifttragenden Monument vorliegen, bleibt unklar, ob der Bezug zu Jes 55,1 o omnes sitientes venite ad quas et qui non habetis argentum properate emite et comedite respektive Joh 7,37 si quis sitit veniat ad me et bibat metaphorischer Natur ist oder einen konkreten Bauzusammenhang aufweist. Der Fundort der Inschrift scheint auf letzteres hinzudeuten. Zur Formulierung de donis Dei et sancti: R. Haensch, Zwei unterschiedliche epigraphische Praktiken: Kirchenbauinschriften in Italien und im Nahen Osten, in: The Epigraphic Cultures of Late Antiquity, hg. von K. Bolle - C. Machado - C. Witschel (Heidelberger Althistorische Beiträge und Epigraphische Studien 60), Stuttgart 2017, 536-554, [hier:] 544-545. Die Wendung fieri rogare, die auch in Nr. 2 auftritt, ist epigraphisch nur selten bezeugt und kennzeichnet in aller Regel Grabinschriften. Außer den beiden hier besprochenen Inschriften gehört noch AE 2014, 528 (Vercellae), wohl aus dem frühen 8.Jh., in einen christlichen Kontext. Die Datierung der übrigen Stücke reicht zurück bis ins 1.Jh.: CIL IV 357 (Pompei); CIL V 4923 = InscrIt X 5, 1148 (Trumplini); CIL V 5602 (Carnago); CIL V 5873 (Mediolanum); CIL V $6896=$ InscrIt XI 1, $116=$ IAugPraetoria 57 (Chatel Argent); CAG 13/5, 2008, p. $598=$ AE 1988, $859=$ AE 1992, 1182 (Arelate).

Resurrection of the Body: A Scholastic Discussion in its Medieval and Modern Contexts, „History of Religions” 30 (1990) fasc. 1, 51-85.

10 So aufgefasst bei ILCV, wo im Kommentar zur betreffenden Nummer weitere christliche Brunneninschriften angeführt werden. 


\subsection{ILCV 3855}

Fund-/Aufbewahrungsort: Roma/Rom, Lateranmuseum

Monument/Datierung: in der linken unteren Ecke einer sehr großen Grabplatte

In no(mine) $\mathrm{D}$ (omi)ni ego Formusanus cond(idi) una cum / coniuge mea Sufua sepulchrum istu(m) / fieri rogabimus pro remedium anim(a)e / n(ost) $\mathrm{r}(\mathrm{a})$ e et si aliouis sepulchru(m) istum / biolare bolueri(t) (h)abea(t) anathema / da patre et filiu et $\mathrm{s}(\mathrm{an}) \mathrm{c}(\mathrm{tu}) \mathrm{m} \mathrm{sp(iritu)m} \mathrm{et} \mathrm{cum} \mathrm{/} \mathrm{Iuda} \mathrm{traditore} \mathrm{(h)abea(t)}$ portione $(\mathrm{m})$

Im Namen des Herrn haben wir, ich Formusanus gemeinsam mit meiner Gattin Sufua, dieses Grab errichtet. Wir haben (dies) zum Heil unserer Seele veranlasst, und wenn jemand dieses Grab schänden will, sei er mit dem Anathem belegt von Vater und Sohn und Heiligem Geist und soll das Schicksal mit dem Verräter Judas teilen.

Kommentar: Die Namen der beiden Verstorbenen, Formusanus und Sufua, sind epigraphisch singulär. Während der erste eine Ableitung aus Formosus darstellt, bleibt der Hintergrund von Sofua offen ${ }^{11}$. Ein Mann namens Sofus ist in CIL II $2842=$ ERPSoria 55 (Las Cuevas de Soria) genannt. Die Wendung pro remedium animae beziehungsweise korrekt pro remedio animae tritt nur noch einmal in einer christlichen Grabinschrift, wohl aus dem Jahr 1048, aus La Cogollina y la Garba in Asturien auf: IHC 276 (p. 2, 98). Fast ebenso sporadisch ist der Ausdruck habere portionem bezeugt. Die insgesamt nur fünf Nachweise, unter diesen hier auch noch Nr. 6, sind allesamt Teil der Formulierung cum Iuda traditore habeat portionem: CIL XI 329 = ILCV 3853 (Ravenna); Epigraphica 41, 1979, 178 = AE 1982, 369 (Dertona); CIL V 307 = InscrIt X 1, 560 = ILCV 3846 (em.) (Pola). Etwas häufiger und vor allem auch in Italien gebräuchlich ist die Wendung cum Iuda (traditore) habeat partem: ICUR VII 18191; ICUR VIII $21396=$ ILCV 3845 = ICaRoma 118; ILCV 1273 (add.); vielleicht auch ILCV 1630 (alle Roma); Camodeca 2017a, p. 87, 1 (Teanum Sidicinum); Lambert 2004, p. 95 (Salernum); eventuell CIL XI 6779 = ILCV 3848 (Ravenna); SRD $949=$ AE 1999, 806 (Peninsola del Sinis); IHC $403=$ ICERV 262 = ILCV 3847 (Carthago Nova); möglicherweise ebenso AE 2008, 1668 (Menzel Yahia-Tafekbsite). Von der iberischen Halbinsel ist parallel die Wendung cum Iu[d]am Iscariot (h)abead participium (CICMerida 45, Emerita) beziehungsweise cum Iud(a) participium (h) abeat (CIL II ${ }^{2}$ 7, 678 = HEp 1989, 252, Corduba) überliefert. Zur Nennung des Judas im Kontext

11 ILCV erwägt, dass der Name Sueva gemeint gewesen sei. 
von Grabstrafen vgl. J. Del Hoyo, Ne velis violare. Imprecaciones contra los profanadores de tumbas, in: El mundo de los difuntos: culto, cofradías y tradiciones, Simposium San Lorenzo del Escorial 4 al 7 de septiembre. Vol. II, hg. von F.J. Campos - F. de Sevilla (Estudios Superiores des Escorial), San Lorenzo del Escorial 2014, 817-822 und insbesondere in iberischen Grabinschriften S. Perea Yébenes, La mención a Judas Iscariota en epitafios latinos cristianos de la Hispania visigoda y bizantina: el delito sepulcral y la condena mágica, „Myrtia” 21 (2006) 235-276.

\subsection{ILCV $3856=$ ICaRoma 121}

Fund-/Aufbewahrungsort: Roma/Rom, Lateranmuseum Monument/Datierung: Marmorplatte, 7./8.Jh.?

(H)ic requiescit in pace dom(i)na Bono/sa q(uae) vix(it) ann(os) XXXXXX et dom(i)no Menna / q(ui) vixit (an)nos [---] e(t) (h)abeat anat(h)/ema a Iuda si quis alterum (h)omine(m) sup / me posueri(t) anathema (h)abeas da tricenti decem et octo patriarche(s) / qui c $\{\mathrm{h}\}$ anon(e)s $\{$ es $\}$ posuerun(t) et da $\mathrm{s}(\mathrm{an}) \mathrm{c}(\mathrm{t})$ a Chr(ist)i / (q)uat(t)uor eugvangelia

Hier ruht in Frieden die Herrin Bonosa, die 60 Jahre alt geworden ist, und Herr Menna, der (?) Jahre gelebt hat. Belegt mit dem Anathem von Judas soll sein, wer einen anderen Menschen über mir bestattet; belegt mit dem Anathem sollst du sein von den 318 Patriarchen, die die Kanones festgelegt haben, und von den vier heiligen Evangelien Christi.

Kommentar: Die Verbreitung des Namens Bonosa zeigt deutliche Schwerpunkte, einerseits in Rom und dem unmittelbaren Umfeld $(n=25)$, ferner in Nordafrika $(n=26)$ sowie im Balkanraum $(n=12)$. Mindestens ein Drittel der betreffenden Inschriften gehören in einen christlichen Kontext $(n=22)$. Der Name Menna ist nur noch ein weiteres Mal aus Salona in einer christlichen Grabinschrift bezeugt: Salona IV 1, 265. Er stellt eine Variante des griechischen Namens Mena oder Menas dar. Menna ist in der dalmatischen Inschrift ebenfalls als dominus charakterisiert. Möglicherweise war er kein Laie, in jedem Fall aber weisen dominus und domina auf übergeordnete Personen. Die Nennung der tricenti decem et octo patriarches nimmt Bezug auf die angebliche Zahl der am Konzil von Nicäa 325 teilnehmenden Bischöfe, während die einschlägige Forschung entsprechend den frühesten Nachrichten von rund 250 Personen ausgeht, vgl. H. Gelzer - H. Hilgenfeld - O. Cuntz (Hrsg.), Patrum Nicaenorum nomina Latine, Graece, Coptice, Syriace, Arabice, Armeniace (Bibliotheca scriptorum 
Graecorum et Romanorum Teubneriana), Leipzig 1995 (Neudruck 1. Auflage 1898) und dazu J. Ulrich, Rezension zu H. Gelzer - H. Hilgenfeld - O. Cuntz (Hrsg.), Patrum Nicaenorum nomina Latine, Graece, Coptice, Syriace, Arabice, Armeniace (Bibliotheca scriptorum Graecorum et Romanorum Teubneriana), Leipzig 1995 (Neudruck 1. Auflage 1898), ZACh 2 (1998) 147-150. Beginnend mit Hilarius von Poitiers, Hil. Syn. 86, sind kanonisch 318 Teilnehmer überliefert, vermutlich angelehnt an die 318 Knechte Abrahams: Gen 14,14 quod cum audisset Abram captum videlicet Loth fratrem suum numeravit expeditos vernaculos suos trecentos decem et octo et persecutus est eos usque Dan, vgl. C. Piétri - L. Piétri (Hrsg.), Das Entstehen der einen Christenheit (250-430) (Die Geschichte des Christentums 2), Freiburg 1996, 305; J. Ulrich, Konstantin der Große und die Frage nach den Vätern des Konzils von Nizäa, in: Väter der Kirche. Ekklesiales Denken von den Anfängen bis in die Neuzeit. Festgabe für Hermann Josef Sieben SJ zum 70. Geburtstag, hg. von J. Arnold - R. Berndt - R.M.W. Stammbacher, Paderborn 2004, 149-165. Die Inschrift verwendet den spezifischen Begriff patriarches, der nicht vor dem 6.Jh. Eingang in die Epigraphik gefunden hat, dazu D. Feissel, L'évêque, titres et fonctions d'après les inscriptions grecques jusqu'au VIIe siècle, in: Actes du XIe congrès international d'archéologie chrétienne. Lyon, Vienne, Grenoble, Genève, Aoste, 21-28 septembre 1986, hg. von N. Duval (Collection de l'École française de Rome 123 / Studi di antichità cristiana 41), Rome 1989, 801-828, [hier:] 806-808. Der Gebrauch der Wendung anstelle von episcopi scheint hier entsprechend datierungsbedingt und nimmt nicht Bezug auf die unterschiedlichen bischöflichen Rangstufen.

\subsection{ILCV 3858a}

Fund-/Aufbewahrungsort: Roma/Rom, S. Maria in Domnica

Monument/Datierung: wegen der Konsuldatierung wohl nicht später als 6.Jh.; vgl. als Parallele etwa CIL V 7793 (Albingaunum)

[---]iqoc[---] / [---]a[---] / [--- co]nss(ulibus) (viris) cc(larissimis) Kal(endis) [---]e[---] / [---]nolqeu Th(e)odora [---] / [---] est in pace mense Martio [---] / [---] si quis coc (!) sepulchrum pos[t mortem meam (?)] / [violav]er[i]t (h)aveat anathema q(u)ei $\{\mathrm{i}\}$ ti [---] / [---]et ante tribunal(em) D(omi)no Ze[su Chr(ist)o] / [Nazar]neu

Späterer Zusatz: fiat fiat [fiat] 
Die zerstörten ersten Zeilen nennen ein konsuldatiertes Grab einer gewissen Theodora, die im März bestattet wurde. Wer dieses Grab nach meinem = des Stifters (?) Tod geschändet haben wird, sei mit dem Anathem belegt; er möge keine Ruhe finden (?), und sich vor dem Gericht unter dem Vorsitz des Herrn Iesus Christus des Nazareners verantworten. Es möge geschehen, geschehen [geschehen].

Kommentar: Für die Wiederholung von fiat am Inschriftenende bietet ILTun 1251 = BE 684 aus dem nordafrikanischen Bulla Regia eine Parallele: Der Türsturz der Alexanderkirche trägt die Schutzinschrift $D(o m i) n(u)_{s} c u$ stodiat introitum tu(u)m / et exitum tuum ex hoc nunc / et usque in saeculum / amen fiat fiat. Sie ist entlehnt von Ps 120,8: Dominus custodiat exitum tuum et introitum tuum amodo et usque in aeternum und wird am Ende durch fiat fiat verstärkt. Entsprechende Wunschformeln, die regelmäßige Verwendung des Konjunktivs und verstärkende finale Begriffswiederholungen sind aus antiken Fluchtafeln gut bezeugt, vgl. im Überblick zu den Grundtypen lateinischer Verwünschungsformeln A. Kropp, Magische Sprachverwendung in vulgärlateinischen Fluchtafeln (defixiones) (ScriptOralia 135. Reihe A 39), Tübingen 2008, 144-160. Vgl. auch W. Speyer, Fluch, RACh VII 1169-1288, [hier:] 1250 und 1263. In den defixiones beschließen häufig die Zeitadverbien cito, iam, $\tau \alpha \chi v ́$ oder $\eta \not \delta \eta$ formelhaft die Texte. Das ihnen inhärente Drängen wird dadurch unterstrichen, dass sie ein- oder mehrmals wiederholt werden und in Kombination stehen, vgl. dazu zuletzt U. Ehmig, Woher nehmen und nicht stehlen? Überlegungen zur Beschaffung und Bearbeitung des Beschreibstoffes für antike Flüche, erscheint in einer Festschrift 2019.

\subsection{ILCV $3866=$ ICaRoma 120}

Fund-/Aufbewahrungsort: Roma/Rom, Santa Caecilia in Trastevere Monument/Datierung: Marmorplatte, 6.Jh.

Hic requiescit in pa/ce Argentia qui bix/it plus minus annos XL lo/cum bero quem sibi benerabi/lis abbatissa Gratiosa prepa/raberat se vibam mihi eum ces/sit coniuro per patrem et fi/lium et spiritum $\mathrm{s}(\mathrm{an}) \mathrm{c}(\mathrm{tu}) \mathrm{m}$ et di/em tremendam iudicii ut nul/lus presumat locum istum / ubi requiesco violare quod / si qui po(s)t (h)anc coniura/tionem presumserit ana/t(h)ema (h)abeat de Iuda et re/pra Naman Syri (h)abeat

Hier ruht in Frieden Argentia, die etwa 40 Jahre alt geworden ist. Den Ort aber, den die verehrungswürdige Äbtissin Gratiosa für sich vorgesehen hat- 
te, den hat sie mir zu Lebzeiten abgetreten. Ich beschwöre durch Vater und Sohn und Heiligen Geist und den fürchterlichen Tag des Gerichtes, auf dass niemand wagt, diesen Ort, wo ich ruhe, zu schänden, denn, wenn er es nach dieser Beschwörung gewagt haben wird, sei er mit dem Anathem von Judas belegt, und er soll die Lepra des Syrers Naman haben.

Kommentar: Die beiden in der Inschrift genannten Namen sind in dieser Form nur vereinzelt bezeugt, auch wenn ihre Ableitung leicht nachvollziehbar ist. Gratiosa ist zwei weitere Male aus Rom (ICUR VIII 21081 und 21111), Argentia dreimal aus Raetia (CIL III $11971=$ IBR $370=$ CSIR D I 1, 364, Castra Regina), Dalmatia (ILJug III 1994, Onaeum) und der Africa proconsularis (CIL VIII $454=11525=$ ILCV 2973h, Ammaedara) belegt. Ebenfalls selten ist die inschriftliche Erwähnung einer abbatissa, wobei die Hälfte der bekannten Exemplare sich in Rom konzentriert: CIL VI 9235 $=$ ILCV 3770 = ICUR I 420; ILCV 1650 (add.) = ICUR VIII $20836=$ AE 1902, 75; ILCV 3866; ICUR II 5734; ICUR V 13670 (p. 416); ICUR VIII 20836 (alle Roma); ferner CIL X 4514 = ILCV 1651 (Casilinum); SRD $997=$ AE 1991, 906 (Carales); CAG 13/3, 2005, p. 175 (Massilia); CIL XII 5352 (p. 856) = ILCV $1652=$ CAG 11/1, 2003, p. 460 (Narbo); IBC 183 (Hackness). Die früheste epigraphische Erwähnung einer abbatissa (ILCV 1650) datiert in das Jahr 514, vgl. H. Emonds, Aebtissin, RACh I, 126128, [hier:] 126. Der Fluch des Aussatzes des Syrers Naaman geht zurück auf die Erzählung in 2. Kön 5; zum Aussatz F.W. Bayer, Aussatz, RACh I, 1023-1028.

\subsection{ILCV 1293 (add.) $=$ IHC $336=$ CICMerida $29=$ ICERV 47}

Fund-/Aufbewahrungsort: Emerita/Mérida (Lusitania), ehemals Straße Alfonso IX Nr. 18, gegenüber der Basilika Santa Eulalia

Monument/Datierung: in vier anpassende Teile zerbrochene Marmortafel von $59 \times 61 \times 2 \mathrm{~cm}$; Buchstabenhöhe 3,5-5 cm, 7.Jh.?

Quisquis conspicis hoc sepul/tur(a)e opus Eolalii clerici confessori / abtus est locus sed si quis / vero hoc monumentum meum / inquietare voluerit sit anathe/ma percussus lebra Gezie / perfruatur et cum Iuda / traditore (h)abeat portio/nem et a leminibus ec(c)lesi(a)e / separetur et a communi/onem s(an)-c(ta)m sec[lusus (a)eter]nis / [---]

Wer auch immer Du dieses Grab betrachtest, es ist der dem Geistlichen und Bekenner Eulalius angemessene Ort. Wenn aber wirklich jemand vorhaben wird, dieses mein Grabmal zu stören, sei er mit dem Anathem belegt und 
möge sich an der Lepra des Gehasi erquicken, und er soll Anteil am Schicksal des Verräters Judas haben und von den Schwellen der Kirche getrennt sein und von der heiligen Gemeinschaft auf ewig (?) ausgeschlossen sein.

Kommentar: Der mit rund 40 Belegen bezeugte Name Eulalius/-a ist in Rom und Italien $(\mathrm{n}=18)$ sowie auf der iberischen Halbinsel $(\mathrm{n}=17)$ konzentriert. Im konkreten Fall ist von einem Bezug zur lokalen Märtyrerin Eulalia, bei deren Basilika die Grabtafel gefunden wurde, auszugehen. Dieselbe Verbreitung wie der Name zeigt der selten gebrauchte Begriff inquietare zur Beschreibung der Grabschändung mit folgenden Belegen: CIL VI 27498; CIL VI 29947 (p. 4044) = ILS 8187 (beide Roma); CIL X 2289 $=$ ILS 8201 (Puteoli); CIL XI 147 = ILS 8241 (Ravenna); CIL II ${ }^{2}$ 7, 678 = HEp 1989, 252 (Corduba); CILA III 2, 586 = HEp 1995, 386 (Carchel); CICMerida 45 (Emerita). Der Aussatz des Gehasi als Fluch in Folge seiner Untreue nach der Heilung des Naaman rekurriert auf 2. Kön 5,27: sed et lepra Naaman adherebit tibi et semini tuo in sempiternum et egressus est ab eo leprosus quasi nix. Die Wendungen limines ecclesiae und communio sancta stehen, so verbreitet sie auch in anderen Schriftenquellen sind, epigraphisch singulär.

\subsection{IRG III $66=$ IHC $138=$ ICERV 188}

Fund-/Aufbewahrungsort: San Pedro de Tomeza (Hispania citerior), 1741 neben dem Atrium der Kirche auf dem alten Friedhof; Museo de Pontevedra

Monument/Datierung: Fragment eines Sarkophagdeckels, erhalten $1,30 \times 0,47 \mathrm{~m}$; Buchstabenhöhe 7,5 cm, 1. Mai 624

Hic requi/[e]scit corpus / Ermengon[tis] / transivit [in] Ca/l(endas) Mai(as) / (a)era DCLXII [---] quiqumq(ue) / hoc legerit et temer(ario) / [aus]u voluerit corpus alien[um] / in loco isto deponere anat(h)ema sit

Hier ruht der Leib der Ermengon, die an den Kalenden des Mais des Jahres 672 der Ära verstorben ist. Wer auch immer dies gelesen haben wird und in unüberlegtem Unterfangen einen anderen Leib an diesem Ort niedergelegt haben wollen wird, sei mit dem Anathem belegt.

Kommentar: Der germanisch-vandalische Name $(\mathrm{H})$ ermengon ist lediglich ein weiteres Mal aus Hippo Regius bezeugt (AE 1951, 267). Der Begriff transire zur Beschreibung des Übergangs vom irdischen Leben ins Himmelreich - CIL XIII $8478=$ ILCV $2919=$ RSK $489=$ IKöln $754=$ 
CLE 772 (Colonia Claudia Ara Agrippinensium) spricht von ad caelesti[a] [reg]na transivi[t] - ist nur noch wenige weitere Male und ausschließlich in christlichem Kontext gebraucht: ILCV 2785 = ICERV $269=$ HEp 1995, 124 = AE 1992, 1081 (Manacor); CIL XIII 2799 = ILCV 1281 (add.) = CLE 2197 (Augustodunum); CIL VIII 20288 = ILCV 3436 = CLE 1834 (Satafis); vgl. dazu M.T. Muñoz García de Iturrospe, Tradición formular y literaria en los epitafios latinos de la Hispania cristiana, (Veleia Series Minor 7), Vitoria Gasteiz 1995, 192-193. Zu der ab dem 5. und bis ins 15. Jh. vor allem auf der iberischen Halbinsel in Inschriften gebrauchten sogenannten spanischen Ära vgl. zuletzt und mit entsprechenden Literaturhinweisen J.M. Abascal, La era consular hispana y el final de la práctica epigráfica pagana, „Lucentum” 19/20 (2000/2001) 269-292 und J. Végh, Inschriftkultur und Christianisierung im spätantiken Hispanien, in: The Epigraphic Cultures of Late Antiquity, hg. von. K. Bolle - C. Machado - C. Witschel, Stuttgart 2017, 82-84.

\subsection{Bibl. Epigr. 772}

Fund-/Aufbewahrungsort: Limonum Pictonum/Poitiers (Aquitania), Rue du Père-de-la-Croix

Monument/Datierung: Hypogäum Mellebaude, rechter Türrahmen, spätes 7./frühes 8 .Jh.

In Dei nomine ego / hic Mellebaudis / reus et servus Ie(su)m Chr(ist)o(m) / institui mihi $\{\mathrm{i}\}$ spe/luncola ista ubi / iacit indigni[---] / sepultura mea [---] / quem feci in nome/ne D(omi)ni Ie(su)m Chr(ist)i quem / amavi in quod / credidi v[ere dig]num / est confetir[i] / viv[um ---] / [cuius glori]a magna est / ubi pax fedis c[ari]/tas est ipse $\mathrm{D}(\mathrm{eu}) \mathrm{s}$ [et] / [ho]mo est et $\mathrm{D}(\mathrm{eu}) \mathrm{s}$ in illo / si quis qui non hic / amat adorare $\mathrm{D}(\mathrm{omi}) \mathrm{n}(\mathrm{u}) \mathrm{m} \mathrm{Ie}(\mathrm{su}) \mathrm{m} / \mathrm{Chr}$ (istu)m et distruit opera / ista sit anathema / maranatha / usquid in sempiternum

Im Namen Gottes habe ich Mellebaude, Schuldner und Sklave Iesu Christi, mir hier diesen kleinen unterirdischen Grabbau errichtet, wo der Unwürdige liegt. Meine Grabstätte, die ich im Namen des Herrn Iesus Christus errichtet habe, den ich geliebt habe, an den ich geglaubt habe, der es wahrlich verdient, als lebendiger (Gott) anerkannt zu werden, dessen Ruhm dort groß ist, wo Friede, Glaube und Liebe sind. Er ist selbst Gott und Mensch, und Gott ist in jenem. Wenn jemand hier es nicht liebt, den Herrn Iesus Christus zu verehren, und diesen Bau zerstört, sei er mit dem Anathem belegt, Maranatha, bis in Ewigkeit. 
Kommentar: Das Hypogäum Mellebaude ist Teil einer 1878 entdeckten Nekropole mit knapp 40 aufgedeckten Grablegen des 7./8. $\mathrm{Jh}$. In den 4,8 $\mathrm{m}$ tiefen und im vorderen Bereich 3,5 $\mathrm{m}$, im hinteren 4,5 m breiten, überkuppelten Raum führten 12 Stufen. Die Tür an ihrem unteren Ende trägt auf der rechten Wange die Inschrift, die sich als Kombination von Bau- und Grabinschrift sowie Glaubensbekenntnis darstellt. Die architektonischen Elemente des Hypogäums sind reich verziert. Eine monographische Studie ist angekündigt (https://cescm. hypotheses.org/3590 und https://journals.openedition.org/adlfi/3160 letzter Aufruf 11.5.2018); vgl. einstweilen A. Grabar, Recherches sur les sculptures de l'Hypogée des Dunes, à Poitiers, et de la Crypte SaintPaul de Jouarre, „Journal des savants” (1974) fasc. 1, 3-43; C. Heitz, L'hypogée de Mellebaude à Poitiers, in: L'Inhumation privilégiée du IVe au VIIIe siècle en Occident. Actes du colloque tenu à Créteil les 16-18 mars 1984, hg. von Y. Duval - J.-C. Picard, Paris 1986, 91-96; B. Palazzo-Bertholon, L'hypogée des Dunes à Poitiers: une lecture archéologique renouvelée, „Bulletin de Liaison” 31 (2007) 60-61. Der Grabstifter Mellebaude ist aus keinen anderen Quellen bezeugt. Die Annahme, es handle sich um einen Abt (vgl. so C. Morris, The Sepulchre of Christ and the Medieval West. From the Beginning to 1600, Oxford 2005, 120) ist nicht verifizierbar. Ein fränkischer König und comes domesticorum namens Mellobaudes ist für die 370er Jahre bei Amm. 30, 3, 7 und 31, 10, 6 überliefert. Während servus Iesu zu den geläufigen epigraphischen Selbstbezeichnungen als Diener Gottes zählt - vgl. Ehmig 2015b -, ist die Benennung als reus singulär. Speluncola wird nur noch in einer weiteren christlichen 1nschrift aus Rom zur Bezeichnung der Grablege verwendet: ICUR IV $12520=$ ILCV 1980 $(\mathrm{em})=$. CLE 911. Die Verfluchung innerhalb der Inschrift si quis qui non hic amat adorare d(omi)n(u)m Ie(su)m Chr(istu)m et distruit opera ista sit anathema maranatha ist einerseits eine sehr enge Ableitung von 1. Kor 16,22: si quis non amat Dominum nostrum Iesum Christum, sit anathema, Maranatha, andererseits mit dem Zusatz distruit opera ista ein eindeutiger Bau- und Grabschutz. Das aramäische Maranatha verstärkt als Bittruf „Unser Herr, komm!“ - ähnlich wie fiat fiat in Nr. 4 - die Verwünschung; zu Maranatha A. Ehrensperger, Akklamationen, liturgische Rufe, https:/www.gottesdienst-ref.ch/perch/resources/0203-01-06-akklamationen-liturgische-rufe-kopie.pdf (letzter Aufruf 12.5.2018) 2005; vgl. auch W. Speyer, Fluch, RACh VII 1169-1288, [hier:] 1264. Die Wendung in sempiternum ist überraschenderweise nur noch zwei weitere Male epigraphisch bezeugt, zum einen in einer weiteren christlichen Grabinschrift aus Rom (CIL X $4530=$ ILCV 2366) 
und zum anderen in einer paganen Grabinschrift eines Veteranen der legio VIII Augusta in Syrien, der sein Grab dedicavit monumentum suum in sempiternum di $\{i\}$ s Manibus suis et Fl(aviae) Titiae uxoris suae inferisque et heredibus suis posterisque eorum und es auf diese Weise vor fremdem Zugriff schützte (CIL III 191 (p. 973) = IGLS II 455, Beroea).

\subsection{CIL XII 5755 = ILCV $3857=$ CAG 4, 1997, p. $211=$ AE 1983, $665=\mathrm{AE} 1995,1036$}

Fund-/Aufbewahrungsort: Reii Apollinaris (Gallia Narbonensis), zwei Fragmente derselben Inschrift: 1. 1869 in Peyruis, in den Fundamenten eines Privathauses an der Route Nationale 96, erhalten ist ein Abklatsch; 2. 1990 in Ganagobie, $4 \mathrm{~km}$ südwestlich von Peyruis, $50 \mathrm{~m}$ östlich des Priorats Notre Dame, Musée de Digne

Monument/Datierung: weiße Marmortafel, 1. Fragment (links) $18,5 \times 18,5 \times 5,6-6,6 \mathrm{~cm} ; 2$. Fragment (rechts) $18,5 \times 16,5 \times 4,3-6,8 \mathrm{~cm}$; Buchstabenhöhe 1-3,8 cm; 7./8.Jh.

Hic requiscit in $\mathrm{p}[\mathrm{ace}] /$ bon(ae) mem(oria)e Iuveldis [quae vi]/xit an(n)us plu(s) menus XXV[---] / si qius (!) sarcofa[go eum] / voluerit remover[e il]/li anatema nove(---) ger[---] / taram(---) si[---] ben(e) req(uiescat?)

Hier ruht in Frieden zum guten Gedenken Iuveldis, die etwa (mindestens) 25 Jahre alt wurde. Wenn jemand den Sarkophag entfernt haben wollen will, sei er mit dem Anathem belegt. (...) Sie möge ruhen.

Kommentar: Die Kombination der Wendungen in pace und bonae memoriae, wie sie auch in Nr. 10 auftritt, ist in Grabinschriften überaus häufig bezeugt. Von den mehr als 750 Belegen konzentrieren sich 300 in Rom und Italien, 230 in der Gallia Narbonensis und Lugdunensis sowie jeweils knapp 100 in Nordafrika sowie auf Sardinien und Sizilien. Der Name der Verstorbenen ist singulär. Auch wenn die durch Überstrich als Wortabkürzungen markierten Buchstaben am Ende der vorletzten und in der letzten Zeile gut lesbar sind, bleibt ihr Sinn fraglich. Zu überlegen wäre, ob hier ein zweiter Verstorbener namentlich genannt wurde. Ausführlich zur Inschrift J. Guyon, De Pyruis à Ganagobie, à la recherche des compléments d'une inscriptions chrétienne des Alpes de Haute-Provence, in: Orbis romanus christianusque ab Diocletiani aetate usque ad Heraclium. Travaux sur l'antiquité tardive rassemblés autour des recherches de Noël Duval (avec sa bibliographie raisonnée), hg. von 
F. Baratte - J.-P. Caillet - C. Metzger (De l'archéologie à l'histoire), Paris $1995,129-145$.

\subsection{CIL XIII 1661 = ILCV 3858 (em.) = CAG 69/1, 2006, p. 123}

Fund-/Aufbewahrungsort: Albigny-sur-Saone(Lugdunensis), Domaine de Bel-Air, in der Stützmauer einer Terrasse, angelehnt an die dominierenden Felsnischen vor dem Haus

Monument/Datierung: Sarkophagfragment, 7.Jh.

In hoc tumolo requiis/cit membri bon(a)e memori(a)e / Audolena(e) bona karetate / sua $\{\mathrm{m}\}$ qui vixit in / pace an(n)us XXXVII qui a / hoc $\{\mathrm{h}\}$ ossa removit a/natema sit ob(iit) VII Kalen/das Ianuarias

In diesem Grab ruhen die Glieder der Audolena zum guten Gedenken und in guter Nächstenliebe zu ihr, die in Frieden 37 Jahre gelebt hat. Wer von hier die Gebeine entfernt, sei mit dem Anathem belegt. Verstorben ist sie am 7. Tag vor den Kalenden des Januars.

Kommentar: Der burgundische Name Audolena, vgl. Pfister 1996, 994, tritt nur noch ein weiteres Mal inschriftlich in der Gallia Narbonensis auf: CIL XII 2105 (Vienna). In Mainz hieß ein mit drei Jahren verstorbenes Kind Audolendis: CIL XIII $7201=$ ILCV 3595a. Die Wendung bona caritate ist singulär. Zur frühchristlichen Bedeutung von caritas als Mutter aller Tugenden und Teil der bona summa neben fides und spes vgl. zuletzt R. Kany, Tugenden und Laster als Gliederungselemente angewandter Ethik im antiken Christentum, in: Gliederungssysteme angewandter Ethik. Ein Handbuch. Nach einem Projekt von Wilhelm Korff, hg. von W. Korff - M. Vogt, Freiburg 2016, 332-355, [hier:] 349-350.

\subsection{AE 1935, 59}

Fund-/Aufbewahrungsort: Theveste/Tebessa (Africa proconsularis), Marktplatz

Monument/Datierung: sekundäre Ritzinschrift auf einer älteren Grabstele des späten 1./frühen 2.Jh. für den im Alter von 30 Jahren nach fünf Dienstjahren verstorbenen miles der legio III Augusta Marcus Canteius $($ CIL VIII 10626 (p. 2731) = $16546=$ ILAlg I 3109) 
(H)abeat anathem(a) os(sa) sint insepulta $\mathrm{s}(\mathrm{an}) \mathrm{ctis}$ euangelis

Er sei mit dem Anathem belegt. Die Gebeine seien unbestattet durch die heiligen Evangelien.

Kommentar: Die Ritzung ist auf der Grabstele eines römischen Soldaten angebracht. Da keine Fotos, Zeichnungen und beschreibende Informationen dazu vorliegen, wo und wie das Graffito auf dem Stein eingeritzt worden war, muss ein möglicher Bezug zur Grabinschrift des Marcus Canteius, also eine „Vergesellschaftung“ der Textzeugnisse, dazu Ehmig, Vergesellschaftetete Schriften (2019), offenbleiben. Die Überlegungen von A. Truillot, Les pierres qui parlent... Inscriptions inédites de la Région de Tébessa, „Bulletin de la société archéologique de Sousse" 21 (1934) 13-24, [hier:] 22-24, sind entsprechend nicht verifizierbar. Bei der einzigen weiteren lateinischen Inschrift, die den Begriff insepultus verwendet, handelt es sich um ein Epitaph, in dem ein potenzieller Grabschänder verflucht wird: male pereat insepultus iaceat non resurgat cum Iuda partem habeat - er möge elend zugrunde gehen, unbestattet bleiben, nicht auferstehen und das Schicksal des Judas teilen (ILCV $3845=$ ICUR VIII $21396=$ ICaRoma 118).

\section{Theologische Beobachtungen zum Einsatz des Begriffs anathema in den Inschriften}

Im Zusammenhang mit der Frage nach dem Einsatz beziehungsweise der Rolle des frühchristlichen Anathems stellen die oben präsentierten elf Inschriften eine überaus frappierende Erscheinung dar. Anathematisiert werden hier potenzielle Grabschänder respektive - wie dies der Text der ersten Inschrift deutlich macht - Menschen, die, wenn auch gegebenenfalls zu Ehren eines bestimmten Heiligen, geweihtes Wasser gegen Geld verkaufen wollen. Eine so definierte Gruppe von Personen, die von den Verfassern der Inschriften mit dem Anathem belegt werden, erscheint auf den ersten Blick insofern problematisch und überraschend, als die altchristlichen literarischen Quellen - darunter vor allem die sogenannten Konzilskanones - sich bei der Verwendung des Begriffs anathema stets am Wort des Paulus

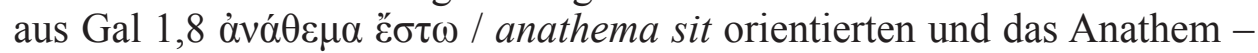
wie der Apostel - ausschließlich über die Irrlehrer verhängten ${ }^{12}$.

12 Ausführlich dazu Zawadzki, Die Anfänge des „,Anathema“ in der Urkirche (2008), 1328-1330; erste Hinweise zu dieser dezidiert antihäretisch orientierten 
Das Anathem kennzeichnet, den literarischen Quellen folgend, einen verfluchten Bereich teuflischen Ursprungs und fungiert als Markierung, die erkennen lässt, welche dogmatischen Positionen als göttlich, das heißt orthodox, gelten dürfen, und welche als diabolisch, das heißt häretisch, $\mathrm{zu}$ verwerfen sind. Der Einsatz des Anathems orientiert sich in diesem Zusammenhang - wie die vor einigen Jahren präsentierte Analyse der hierfür maßgeblichen frühchristlichen Texte ans Licht bringen konnte ${ }^{13}$ - an einem zweifachen Kriterium: Anathematisiert werden nur solche Personen, die hartnäckig an einer unorthodoxen Lehre festhalten und diese auch aktiv verbreiten. Jenes zweifache Kriterium der Hartnäckigkeit und des aktiven Engagements bei der Verbreitung einer antichristlichen Doktrin stellt den festen Rahmen dar, innerhalb dessen sich die Sanktion des Anathems in der antiken christlichen Literatur bewegt. Mit dem Anathem belegt werden demnach keine ,gewöhnlichen“ Sünder, die sich etwa eines moralischen Vergehens schuldig machen, sondern Menschen, die sich frei entscheiden, einer ,verfluchten“, das heißt mit dem christlichen Glauben unvereinbaren Lehre zu folgen, um sie zugleich auch nach außen zu verkünden.

Vor diesem Hintergrund wird deutlich, dass die in den vorgestellten Inschriften anathematisierten Grabschänder, die ja ex definitione keine hartnäckigen und falsche Dogmen verkündenden Irrlehrer sind, den literarischen Quellen zufolge im Grunde genommen gar nicht anathematisiert werden dürften: Eine Grabschändung - auch wenn sie zweifelsfrei ein schweres moralisches Fehlverhalten darstellt - erfüllt die oben beschriebenen Kriterien, die den Einsatz des Anathems rechtfertigen, sicherlich nicht. Warum richten die Verfasser der analysierten Inschriften nun trotzdem ihr Anathem gegen die Grabschänder?

Zwei alternative Antworten bieten sich hier an: (1) Womöglich verwenden die Verfasser der Inschriften den Begriff anathema unkorrekterweise als Synonym für den Ausdruck excommunicatio. Die altkirchliche Exkommunikation stellt nämlich eine Bußmaßnahme dar, mit der in erster Linie Menschen belegt werden, die sich eines schweren moralischen Vergehens schuldig machen; sie bewirkt einen zeitweiligen Ausschluss des Exkommunizierten aus der Gemeinschaft der Gläubigen und gibt ihm so die Möglichkeit, sein falsches Verhalten zu überdenken und zu ändern' ${ }^{14}$. Die anathematisierten Grabschänder würden somit von den Autoren der

Anathematisierungspraxis der Alten Kirche bereits bei A. Seitz, Die Heilsnotwendigkeit der Kirche nach der altkirchlichen Literatur, Freiburg 1903, 117-153.

13 Vgl. hierzu Zawadzki, Die Anfänge des „Anathema“ in der Urkirche (2009); Zawadzki, Die Anfänge des „Anathema “ in der Urkirche (2010).

14 Dazu Zawadzki, Die Anfänge des „Anathema “ in der Urkirche (2009), 517-519. 
Inschriften exkommuniziert und damit zur Umkehr aufgerufen. (2) Nicht auszuschließen aber ist, dass die Texte der Inschriften hier doch der eigentlichen Bedeutung des Terminus anathema folgen. Dies würde bedeuten, dass die Verfasser der Inschriften die von ihnen anathematisierten Grabschänder bewusst in eine Reihe mit den ,verfluchten“ Häretikern stellen, um auf diese Weise alle potenziellen Grabschändungen als eine Art „Häresie“ darzustellen, die gebührend nur mit dem Anathem gebrandmarkt werden kann ${ }^{15}$. Das Anathem soll in diesem Zusammenhang darauf hinweisen, dass eine Grabschändung eine teuflische Tat darstellt, die denjenigen, der sie begeht, als verflucht und verdammt bezeichnen lässt. Das gegen die Grabschänder gerichtete Anathem soll so offenbar eine Abschreckungsfunktion erfüllen und die Leser der Inschriften eindringlich davor warnen, die Ruhe der Toten zu stören.

Offensichtlich divergiert die in den literarischen Texten fassbare Definition des Anathems und sein Verständnis, wie es in den Inschriften zutage tritt. Um dies zu erklären, sind nochmals insbesondere sowohl der zeitliche Horizont der Inschriften, wie auch die Charakteristik ihrer Stifter zu berücksichtigen: Soweit Datierungskriterien - Konsul- und Ehrenangaben, Hinweise aufgrund von stilistischen Merkmalen der Schrift respektive des schrifttragenden Artefakts sowie des archäologischen Kontextes - vorliegen, deuten diese in das 6.-8.Jh., zum Teil eventuell auch noch später. Die anathema-Inschriften sind damit mindestens 200, häufig wohl aber 400 Jahre jünger als jene Texte aus dem frühen Christentum, in denen der Begriff erstmals in Erscheinung tritt und in seiner antihäretischen Verwendung definiert wird ${ }^{16}$. Im Hinblick auf die Stifter der neun Grabinschriften (Nr. 2-10) fällt auf, dass lediglich die Marmortafel aus Emerita (Nr. 6) von einem Geistlichen - clericus confessor - in Auftrag gegeben worden war. Bemerkenswert ist ansonsten der hohe Anteil von Frauen, die allein (Nr. 4, 5, 7, 9, 10) oder gemeinsam mit ihrem Partner (Nr. 2, 3) als Erbauer und Grabinhaber in Erscheinung treten. Auffällig sind in diesem Zusammenhang auch die germanischen - und nicht wie zu erwarten römischen - Namen. Inwieweit hier bestimmte Personengruppen mit der Anathematisierung potenzieller Grabschänder angesichts womöglich prekärer Lebensumstände besondere Vorsichtsmaßnahmen ergriff, müsste in einer umfassenden Analyse frühchristlicher Grabstifter untersucht wer-

15 Die Annahme, dass die Grabschänder von den Autoren der Inschriften tatsächlich als Häretiker betrachtet werden, bestätigt bemerkenswerterweise Inschrift Nr. 3, in der auf die Konzilsväter von Nicäa und die von ihnen gegen die Häresie des Arius erlassenen Kanones Bezug genommen wird.

$16 \mathrm{Zu}$ den Anhaltspunkten für die historische Entwicklung des Anathems Zawadzki, Die Anfänge des ,,Anathema “ in der Urkirche (2008), 1329-1330. 
den. Ungeachtet dessen aber wird deutlich, dass im Laufe der Zeit eine Popularisierung der ursprünglichen Anathematisierungspraxis einsetzt.

$\mathrm{Zu}$ beachten ist, dass einige der besprochenen Inschriften konkrete Hinweise darauf liefern, wie das über die Grabschänder verhängte Anathem inhaltlich verstanden werden soll. Auffallend ist in diesem Zusammenhang zunächst die Tatsache, dass etliche Inschriften (Nr. 2, 3, 5, 6) den Begriff anathema mit dem Verräter Judas in Verbindung bringen. Das Schicksal des Selbstmörders Judas, von dem Jesus sagt, dass es für ihn besser wäre, er sei nie geboren worden (Mt 26, 24), wird laut den Texten der Inschriften das Schicksal aller Grabschänder sein. Das Anathem macht somit aus jedem Grabschänder einen Verräter des Erlösers selbst und weist gleichzeitig darauf hin, dass die Existenz eines jeden Grabschänders eine in jeder Hinsicht verlorene ist. Der anathematisierte Grabschänder steht wie Judas unter dem direkten Einfluss des Satans (vgl. Lk 22,3; Joh 13,27) und wird deshalb - wie der Satan - endgültig der Vernichtung anheimfallen. Interessant erscheint in diesem Zusammenhang die in der Inschrift Nr. 6 gebotene Beschreibung der konkreten Wirkung des Anathems: Der anathematisierte Grabschänder soll aus der Kirche und aus der Gemeinschaft der Heiligen auf ewig ausgeschlossen sein. So soll ihm jede Möglichkeit, an der erlösenden Heilstat Christi teilzuhaben, verwehrt bleiben. Das Anathem bewirkt demnach hier die ewige Verdammnis des Anathematisierten. Auch wenn die altchristliche Literatur keine so scharf profilierten Aussagen über die eschatologische Zukunft der Anathematisierten bietet ${ }^{17}$, kann allein die Tatsache, dass das Anathem in den literarischen Quellen häufig als Hinweis auf den verfluchten Wirkungsbereich des Teufels fungier ${ }^{18}$, dahin interpretiert werden, dass eine Anathematisierung durchaus mit dem Urteil der ewigen Verdammnis beziehungsweise mit dem Wunsch, dass der Anathematisierte - wie der Teufel - der ewigen Verdammnis anheimfallen möge, gleichbedeutend ist. Wenn nun der Autor der genannten Inschrift das Anathem eindeutig in diesem endgültigen Sinne verwendet, bewegt er sich damit durchaus im inhaltlichen Rahmen der altkirchlichen Vorstellung von der Funktion des Anathems.

Abschließend sei noch eine philologische Beobachtung angemerkt. Es fällt auf, dass einige der besprochenen Inschriften (Nr. 1, 6-11) den Ausdruck anathema mit sit konstruieren und damit dem oben zitierten

17 Auch wenn etwa Ignatius von Antiochien in Epistula ad Ephesios 16, 2 behauptet, dass jeder Irrlehrer ,in das unauslöschliche Feuer wandern wird“, vertritt er dennoch in Epistula ad Smyrnenses 4, 1 die Meinung, dass Christus die Macht habe, sogar den hartnäckigsten Häretiker zur Umkehr zu bringen. Hierzu Zawadzki, Die Anfänge des „Anathema “ in der Urkirche (2010), 737-738. Eine prinzipielle Möglichkeit, die eschatologische Erlösung zu erlangen, wird somit von Ignatius niemandem verwehrt.

18 Zawadzki, Die Anfänge des ,Anathema“ in der Urkirche (2009), 500-501. 
Pauluswort aus Gal 1,8 treu folgen, während andere Inschriften (Nr. 2-5) die Formulierung anathema habeat beziehungsweise in direkter Anrede anathema habeas bevorzugen. Die letztgenannte Wendung, die potenzielle Grabschänder „das Anathem haben“ lässt, stellt eine sprachliche Neuerung dar, die vom ursprünglichen und vor allem in den Konzilskanones belegten

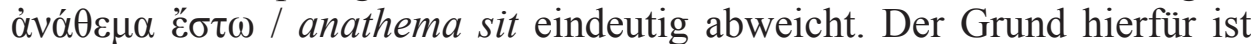
womöglich darin $\mathrm{zu}$ suchen, dass der paulinische Ausdruck anathema sit, wörtlich ,möge er der Fluch sein!“”, den Verfassern der Inschriften sprachlich auf Anhieb nicht (mehr) verständlich erschien, sodass sie sich schließlich entschieden, die ein wenig einfachere Variante anathema habeat, „möge er den Fluch haben!“ zu verwenden.

\section{Fazit}

Die hier besprochenen lateinischen Inschriften mit dem Begriff anathema lassen erkennen, dass die in der altchristlichen Literatur ausschließlich auf die Irrlehrer bezogene Sanktion des Anathems in der ausgehenden Spätantike beziehungsweise im frühen Mittelalter neue Anwendungsbereiche findet. Die ursprünglich anathematisierbare - nur aus den Häretikern bestehende - Personengruppe wird aus der neuen Perspektive um Grabschänder erweitert. Das Anathem kann damit nicht mehr nur den dogmatischen, sondern auch moralischen Bereich des Lebens eines Menschen betreffen. In dieser Hinsicht sind die Inschriften ein zentrales und sprechendes Zeugnis dafür, dass die Sanktion einem Wandel unterworfen war, der schließlich zur deutlichen Verschiebung ihres ursprünglichen - von Paulus definierten - Wirkungsfeldes führte.

\section{Anatema w lacińskich inskrypcjach}

(streszczenie)

Ukończone niedawno badania nad chrześcijańskimi epitafiami zawierającymi terminy adiuro lub coniuro zwracaja uwage na to, że owe epitafia - mające na celu chronić groby przed profanacją - grożą klątwą każdemu, kto ośmieliłby się naruszyć miejsce pochówku zmarłych. Używane w tym kontekście groźby i sankcje karne niejednokrotnie zapożyczano ze Starego lub Nowego Testamentu. Profanacje grobów podlegały między innymi anatemie skazującej profanatorów na karę wiecznego potępienia. Konrad F. Zawadzki, który w ostatnich latach intensywnie zajmował się użyciem terminu ,anatema” w źródłach literackich, zwrócił uwagę na to, że sankcja anatemy we wczesnej literaturze chrześcijańskiej stosowana była przede wszystkim w kontekście sporów dogmatycznych z heretykami. 
Użycie terminu ,anatema” w łacińskich inskrypcjach, które do tej pory nie było jeszcze nigdy przedmiotem badań naukowych, a które w niniejszym przedłożeniu poddane jest szczegółowej analizie, ukazuje, że kara anatemy - skierowana przeciwko profanatorom grobów - dotyczyć mogła w pierwszej linii nie odstępstw dogmatycznych, ale przewinień moralnych. Prezentowane i omawiane w artykule jedenaście inskrypcji łacińskich zawierających termin , ,anatema” oraz datowanych na czas między VI a VIII wiekiem jest wymownym dowodem na to, że sankcja anatemy - początkowo stosowana jedynie w kontekście dogmatycznym - z biegiem lat zaczęła obejmować nowe „przeklęte” obszary.

Słowa kluczowe: anatema; łacińskie inskrypcje; klątwa

\section{Anathema in lateinischen Inschriften}

\section{(Zusammenfassung)}

Eine vor kurzem abgeschlossene Studie zur Verwendung der Begriffe adiuro und coniuro in frühchristlichen Epitaphien machte deutlich, dass jene Epitaphien, derer Ziel es war, potenzielle Grabschänder abzuschrecken, über jeden, der die Ruhe der Toten zu stören wagte, drastische Strafmaßnahmen verhängten. Die in diesem Zusammenhang verwendeten Fluchformeln stammten nicht selten aus dem Alten und Neuen Testament. Grabschändungen wurden unter anderem mit dem Anathem belegt, das zur ewigen Verdammnis des Anathematisierten führen sollte. Konrad F. Zawadzki, der sich neuerdings intensiv mit dem Begriff ,,anathema“ in den literarischen Quellen beschäftigte, wies darauf hin, dass die Sanktion des Anathems in der frühchristlichen Literatur in erster Linie im Zusammenhang mit dem Kampf gegen die Häretiker eingesetzt wurde. Die bisher noch nicht untersuchte - Verwendung des Ausdrucks ,anathema“ in lateinischen Inschriften, die in dieser Studie eingehend analysiert wird, lässt erkennen, dass das gegen die Grabschänder gerichtete Anathem nicht mehr nur dogmatische, sondern auch moralische Verfehlungen betreffen konnte. Die im vorliegenden Beitrag präsentierten und besprochenen elf lateinischen Inschriften, die in die Zeit zwischen dem 6. und 8. Jahrhundert datiert werden, stellen ein klares Zeugnis dafür dar, dass die Sanktion des Anathems im Laufe der Zeit neue ,verfluchte“ Anwendungsbereiche fand.

Schlüsselwörter: anathema; lateinische Inschriften; Fluch

\section{Anathema in Latin Inscriptions}

\section{(abstract)}

A recently finished study of early Christian epitaphs that contain the term adiuro or coniuro to protect the tombs shows that ignoring these instructions would be penalized with a series of draconian punishments. Relevant curses and punitive formulas were borrowed from the Old and New Testaments. Inter alia the culprit should be punished by means of anathema, the eternal damnation. In recent years Konrad F. Zawadzki has intensively dealt with anathema in the literary sources of the early church and pointed out that the punishment was used in doctrinaire disputes against heretics. The use of the term in Latin 
inscriptions, which has not been studied so far, is the focus of this study. The eleven relevant inscriptions dating from the 6th to the 8th century are analyzed epigraphically and theologically. They are directed against moral offenses and point to a later expansion and popularization of the meaning of anathema.

Keywords: anathema; Latin inscriptions; curse

\section{Bibliographie:}

\section{Quellen}

Ignatius Antiochenus, Epistula ad Ephesios, éd. P.T. Camelot, Ignace d'Antioche. Polycarpe de Smyrne. Lettres. Martyre de Polycarpe, SCh 10, Paris 1945, 56-79.

Ignatius Antiochenus, Epistula ad Smyrnenses, éd. P.T. Camelot, Ignace d'Antioche. Polycarpe de Smyrne. Lettres. Martyre de Polycarpe, SCh 10, Paris 1945, 154-156.

\section{Sekundärliteratur}

Abascal J.M., La era consular hispana y el final de la práctica epigráfica pagana, „Lucentum" 19/20 (2000/2001) 269-292.

Bayer F.W., Aussatz, RACh I, 1023-1028.

Bynum C.W., Material Continuity, Personal Survival, and the Resurrection of the Body: A Scholastic Discussion in its Medieval and Modern Contexts, „History of Religions” 30 (1990) fasc. 1, 51-85.

Bynum C.W., The Resurrection of the Body in Western Christianity 200-1336 (Lectures on the History of Religions 15), New York 1995.

Creaghan J.S., Violatio sepulcri. An Epigraphical Study, Princeton 1951.

Ehmig U., Ausschlußverfahren. Eine Gruppe italischer Grabinschriften als Beispiel sozialer Überassimilierung in der römischen Kaiserzeit, „Epigraphica” 77 (2015) 193-205.

Ehmig U., Servus dei und verwandte Formulierungen in lateinischen Inschriften, in: Social Status and Prestige in the Graeco-Roman World, hg. von A.B. Kuhn, Stuttgart 2015, 303-314.

Ehmig U., Woher nehmen und nicht stehlen? Überlegungen zur Beschaffung und Bearbeitung des Beschreibstoffes für antike Flüche, erscheint in einer Festschrift 2019.

Ehrensperger A., Akklamationen, liturgische Rufe, in: https:/www.gottesdienst-ref.ch/ perch/resources/02-03-01-06-akklamationen-liturgische-rufe-kopie.pdf (letzter $\mathrm{Au}$ fruf 12.5.2018).

Emonds H., Aebtissin, RACh I, 126-128.

Feissel D., L'évêque, titres et fonctions d'après les inscriptions grecques jusqu'au VIIe siècle, in: Actes du XIe congrès international d'archéologie chrétienne. Lyon, Vienne, Grenoble, Genève, Aoste, 21-28 septembre 1986, hg. von N. Duval (Col- 
lection de l'École française de Rome 123 / Studi di antichità cristiana 41) Rome 1989, 801-828.

Gelzer H. - Hilgenfeld H. - Cuntz O. (Hrsg.), Patrum Nicaenorum nomina Latine, Graece, Coptice, Syriace, Arabice, Armeniace (Bibliotheca scriptorum Graecorum et Romanorum Teubneriana), Leipzig 1995 (Neudruck 1. Auflage 1898).

Grabar A., Recherches sur les sculptures de l'Hypogée des Dunes, à Poitiers, et de la Crypte Saint-Paul de Jouarre, ,Journal des savants” (1974) fasc. 1, 3-43.

Guyon J., De Pyruis à Ganagobie, à la recherche des compléments d'une inscriptions chrétienne des Alpes de Haute-Provence, in: Orbis romanus christianusque ab Diocletiani aetate usque ad Heraclium. Travaux sur l'antiquité tardive rassemblés autour des recherches de Noël Duval (avec sa bibliographie raisonnée), hg. von F. Baratte - J.-P. Caillet - C. Metzger (De l'archéologie à l'histoire), Paris 1995, 129-145.

Haensch R., Zwei unterschiedliche epigraphische Praktiken: Kirchenbauinschriften in Italien und im Nahen Osten, in: The Epigraphic Cultures of Late Antiquity, hg. von K. Bolle - C. Machado - C. Witschel (Heidelberger Althistorische Beiträge und Epigraphische Studien 60), Stuttgart 2017, 536-554.

Hausmair B., Am Rande des Grabs. Todeskonzepte und Bestattungsritual in der frühmittelalterlichen Alamannia, Leiden 2015.

Heitz C., L’hypogée de Mellebaude à Poitiers, in: L'Inhumation privilégiée du IVe au VIIIe siècle en Occident. Actes du colloque tenu à Créteil les 16-18 mars 1984, hg. von Y. Duval - J.-C. Picard, Paris 1986, 91-96.

Hofmann K., Anathema, RACh I, 427-430.

Del Hoyo J., Ne velis violare. Imprecaciones contra los profanadores de tumbas, in: El mundo de los difuntos: culto, cofradias y tradiciones, Simposium San Lorenzo del Escorial 4 al 7 de septiembre. Vol. II, hg. von F.J. Campos - F. de Sevilla (Estudios Superiores des Escorial), San Lorenzo del Escorial 2014, 809-824.

Iluk J., Amendes sépulcrales dans les épitaphes de l'époque de l'Empire Romain, Danzig 2013.

Kany R., Tugenden und Laster als Gliederungselemente angewandter Ethik im antiken Christentum, in: Gliederungssysteme angewandter Ethik. Ein Handbuch. Nach einem Projekt von Wilhelm Korff, hg. von W. Korff - M. Vogt, Freiburg 2016, 332-355.

Kaufmann C.M., Handbuch der altchristlichen Epigraphik, Freiburg 1917.

Kropp A., Magische Sprachverwendung in vulgärlateinischen Fluchtafeln (defixiones) (ScriptOralia 135. Reihe A 39), Tübingen 2008.

Morris C., The Sepulchre of Christ and the Medieval West. From the Beginning to 1600, Oxford 2005.

Muñoz García de Iturrospe M.T., Tradición formular y literaria en los epitafios latinos de la Hispania cristiana (Veleia Series Minor 7), Vitoria Gasteiz 1995.

Palazzo-Bertholon B., L’hypogée des Dunes à Poitiers: une lecture archéologique renouvelée, „Bulletin de Liaison” 31 (2007) 60-61. 
Perea Yébenes S., La mención a Judas Iscariota en epitafios latinos cristianos de la Hispania visigoda y bizantina: el delito sepulcral y la condena mágica, „Myrtia” 21 (2006) 235-276.

Pietri C., La Bible dans l'épigraphie de l'Occident latin, in: Le monde latin antique de la Bible, hg. von J. Fontaine - C. Pietri, Paris 1985, 189-205.

Piétri C. - Piétri L. (Hrsg.), Das Entstehen der einen Christenheit (250-430) (Die Geschichte des Christentums 2), Freiburg 1996.

Seitz A., Die Heilsnotwendigkeit der Kirche nach der altkirchlichen Literatur, Freiburg 1903.

Speyer W., Fluch, RACh VII, 1169-1288.

Truillot A., Les pierres qui parlent... Inscriptions inédites de la Région de Tébessa, „Bulletin de la société archéologique de Sousse" 21 (1934) 13-24.

Ulrich J., Rezension zu H. Gelzer - H. Hilgenfeld - O. Cuntz (Hrsg.), Patrum Nicaenorum nomina Latine, Graece, Coptice, Syriace, Arabice, Armeniace (Bibliotheca scriptorum Graecorum et Romanorum Teubneriana), Leipzig 1995 (Neudruck 1. Auflage 1898), Zeitschrift für Antike und Christentum 2, 1998, 147-150.

Ulrich J., Konstantin der Große und die Frage nach den Vätern des Konzils von Nizäa, in: Väter der Kirche. Ekklesiales Denken von den Anfängen bis in die Neuzeit. Festgabe für Hermann Josef Sieben SJ zum 70. Geburtstag, hg. von J. Arnold - R. Berndt R.M.W. Stammbacher, Paderborn 2004, 149-165.

Végh, J., Inschriftkultur und Christianisierung im spätantiken Hispanien, in: The Epigraphic Cultures of Late Antiquity, hg. von. K. Bolle - C. Machado - C. Witschel, Stuttgart 2017, 82-84

Zawadzki K.F., Die Anfänge des ,Anathema“ in der Urkirche. Teil 1: Status quaestionis, VoxP 28 (2008) 1323-1334.

Zawadzki K.F., Die Anfänge des „Anathema“ in der Urkirche. Teil 2: Anhaltspunkte für das Anathem im Neuen Testament, VoxP 29 (2009) 495-520.

Zawadzki K.F., Die Anfänge des „Anathema“ in der Urkirche. Teil 3: Anathematisierungspraxis in den Schriften der Apostolischen Väter und im apokryphen Korintherbrief, VoxP 30 (2010) 721-766. 
